\title{
Formation of $\mathrm{GaP} / \mathrm{Si}(100)$ heterointerfaces in presence of inherent reactor residuals
}

\author{
Oliver Supplie, ${ }^{*, \dagger, \uparrow, \uparrow}$ Matthias M. May, ${ }^{\dagger, \ddagger}$ Christian Höhn, ${ }^{\ddagger}$ Helena Stange, ${ }^{\ddagger}$ \\ Antonio Müller, ${ }^{\dagger}$ Peter Kleinschmidt, ${ }^{\dagger}$ Sebastian Brückner, ${ }^{\dagger, \ddagger}$ and Thomas \\ Hannappel ${ }^{\dagger, \ddagger}$
}

Technische Universität Ilmenau, Institut für Physik, 98693 Ilmenau, Germany, Helmholtz-Zentrum Berlin, Institute for Solar Fuels, 14109 Berlin, Germany, and Humboldt Universität zu Berlin, Institut für Physik, 12489 Berlin, Germany

E-mail: oliver.supplie@tu-ilmenau.de

\begin{abstract}
Adequate silicon preparation is a prerequisite for defect-free III-V growth on Si. We transfer the silicon processing from clean to GaP containing metalorganic vapor phase epitaxy reactors, where we monitor the entire process in situ with reflection anisotropy spectroscopy and analyze the chemical composition of the surface with Xray photoelectron spectroscopy. Beyond a certain sub-monolayer threshold value of $(\mathrm{Ga}, \mathrm{P})$ residuals found on the $\mathrm{Si}(100)$ surface, GaP grows with an inverted majority sublattice. Analogously to III-V growth on two-domain substrates, the co-existence of $\mathrm{Si}-\mathrm{Ga}$ and $\mathrm{Si}-\mathrm{P}$ interfacial bonds at terraces of the same type causes anti-phase disorder in GaP epilayers.
\end{abstract}

${ }^{*}$ To whom correspondence should be addressed

${ }^{\dagger}$ Technische Universität Ilmenau, Institut für Physik, 98693 Ilmenau, Germany

${ }^{\ddagger}$ Helmholtz-Zentrum Berlin, Institute for Solar Fuels, 14109 Berlin, Germany

`Humboldt Universität zu Berlin, Institut für Physik, 12489 Berlin, Germany 


\section{Keywords}

Heterointerfaces, III-V on Silicon, optical in situ spectroscopy, photoelectron spectroscopy 
The integration of III-V and silicon is highly desired in both microelectronics and photovoltaics. Adequate $\mathrm{Si}(100)$ surface preparation is decisive for the quality of subsequently grown GaP epilayers, which could serve as pseudomorphic III-V/Si(100) quasisubstrates. Single-domain $\mathrm{Si}(100)$ surfaces, for example, are a prerequisite for anti-phase domain free III-V heteroepitaxy. ${ }^{1} \mathrm{Si}(100)$ surface preparation has been studied in great detail: When prepared in $\mathrm{H}_{2}$ ambient, monohydride-terminated $\mathrm{Si}$ dimers form at the $\mathrm{Si}(100)$ surface. $^{2}$ Their orientation corresponds to the step structure at the surface: The dimer orientation with respect to the step edges is changing from parallel (B-type, $(2 \times 1))$ to perpendicular (A-type, $(1 \times 2))$ at adjacent terraces seperated by steps of odd numbered atomic heights. ${ }^{3}$ A preference for energetically unfavorable ${ }^{4}$ A-type $\operatorname{Si}(100)$ with $2^{\circ}$ misorientation towards [011], however, could be explained by kinetics: The anisotropic diffusion of Si vacancies preferably along dimers rows and their annihilation at the step edges creates A-type terraces. ${ }^{5}$ Temperatures of about $750{ }^{\circ} \mathrm{C}$ and high $\mathrm{H}_{2}$ pressures are essential here. ${ }^{5}$ Identical conditions applied to almost exactly oriented $\mathrm{Si}(100)$ with larger terraces, in contrast, causes layer-by-layer Si removal, ${ }^{6}$ which needs to be avoided in order to prepare smooth, singledomain A-type surfaces. We recently studied GaP nucleation on $\mathrm{Si}(100)$ and suggested a kinetically limited formation of abrupt heterointerfaces with either $\mathrm{Si}-\mathrm{P}$ or $\mathrm{Si}-\mathrm{Ga}$ bonds depending on the (Ga,P) chemical potential during nucleation. ${ }^{7}$ For GaP grown on $\mathrm{Si}(100)$ in P-rich conditions, we could directly evidence the existence of $\mathrm{Si}-\mathrm{P}$ bonds with X-ray photoelectron spectroscopy (XPS). ${ }^{8}$ Though III-V residuals are inherently present in a realistic processing ambient, $\mathrm{Si}(100)$ studies were mainly performed in clean metalorganic vapor phase epitaxy (MOVPE) reactor conditions. The impact of reactor resiudals on the formation of the heterointerface is still unclear.

Here, we will discuss the crucial influence of surface misorientation and sub-monolayer coverages of $(\mathrm{Ga}, \mathrm{P})$ residuals on the $\mathrm{Si}(100)$ preparation and subsequent GaP nucleation in MOVPE ambient (Aixtron AIX-200). We apply reflection anisotropy spectroscopy (RAS, LayTec EpiRAS-200) throughout processing and benchmark these optical in situ signals to 
in situ mass spectrometry (MS, Hiden HAL301) as well as low energy electron diffraction (LEED, Specs ErLEED 100-A) and XPS (Specs Focus 500 and Phoibos 100) accessible after contamination-free sample transfer. ${ }^{9}$ RAS measures here the difference in complex reflection along [011] and [011] and is particularly sensitive to (100) surfaces of cubic crystals. ${ }^{10}$ Throughout the discussion, we will focus on $\mathrm{Si}(100)$ with $0.1^{\circ} \pm 0.05^{\circ}$ misorientation towards [011]. Important differences compared to higher misorientations will be discussed. We varied reactor pre-conditioning and $\mathrm{Si}$ preparation to control the amount of $(\mathrm{Ga}, \mathrm{P})$ residuals at the Si(100) surface: (1) Samples prepared in a GaP reactor, which was baked at least 30 min at $1010^{\circ} \mathrm{C}$ in $\mathrm{H}_{2}$ and eventually coated with $\mathrm{Si}$, are marked with the index clean and (2) samples prepared in presence of intentionally higher amounts of residual (Ga,P) species(e.g. by reducing the bakeout time and applying quicker pressure ramps after Si homoepitaxy) are indexed with cont. Identical process parameters were applied for thermal deoxidation and Si homoepitaxy with silane $\left(\mathrm{SiH}_{4}\right){ }^{2}$ In case of $\mathrm{GaP} / \mathrm{Si}$ samples, subsequent tertbutylphosphine (TBP) and triethylgallium (TEGa) pulses were offered at $420^{\circ} \mathrm{C}$ prior to pseudomorphic growth of about $40 \mathrm{~nm} \mathrm{GaP.}{ }^{7}$

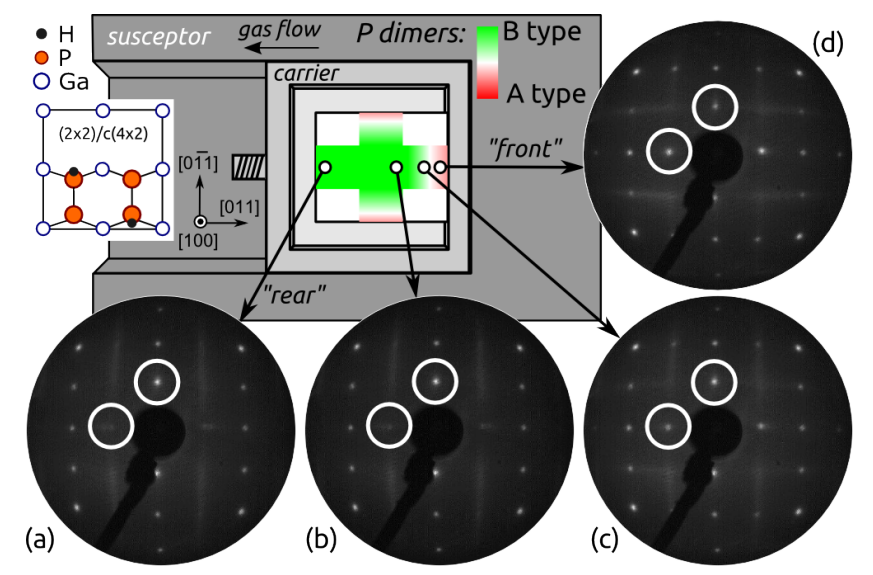

Figure 1: LEED patterns $(\mathrm{E}=102 \mathrm{eV})$ of $(2 \times 2) / c(4 \times 2)$ reconstructed $\mathrm{GaP} / \mathrm{Si}_{\text {clean }}$ and qualitative sketch of the sample (length $\approx 2 \mathrm{~cm}$ ) with color-coded domain imbalance along a scan parallel $(a, b, c, d)$ and perpendicular to the flow direction.

The "P-rich" $(2 \times 2) / c(4 \times 2)$ surface reconstruction of $\mathrm{GaP}(100)$ is formed by buckled $\mathrm{P}$ dimers, which are stabilized by one $\mathrm{H}$ atom each. ${ }^{11,12}$ Its LEED patterns consist of spots 
at half-order (from the $(2 \times 1)$-like $\mathrm{P}$ dimers) and streaks, both in parallel to the $\mathrm{P}$ dimer axis. Due to the zincblende crystal structure, the antiphase domain content at $\mathrm{GaP} / \mathrm{Si}(100)$ surfaces is reflected in domains of mutually perpendicular $\mathrm{P}$ dimers. ${ }^{13}$ Fig. 1 shows LEED patterns of $(2 \times 2) / c(4 \times 2)$ reconstructed $\mathrm{GaP} / \mathrm{Si}_{\text {clean }}$. At about the center of the sample, the $\mathrm{GaP} / \mathrm{Si}(100)$ surface is almost single-domain B-type as indicated by spots at half order along [011] in Fig 1(b). These LEED patterns do not change significantly over large areas of the sample. Towards the front edge, however, the spots at half order along [011] increase in intensity (cf. Fig 1(c)) and at the very edge, the A-type domain even prevails (cf. Fig 1(d)). Similar behaviour is observed at the top and bottom edges. At the rear, in contrast, the B-type majority domains persist (cf. Fig 1(a)). These findings clearly point to an effect at edges close to the susceptor. Two possible explanations are that (i) already the corresponding domain ratio at the $\mathrm{Si}(100)$ surface is affected analogoulsy, or (ii) that diffusion of residual atoms influences the chemical ambient during GaP nucleation.
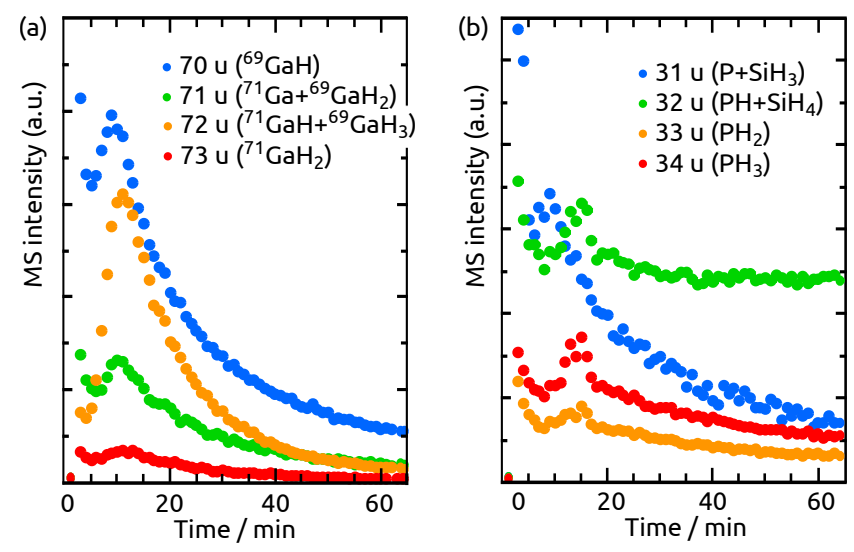

Figure 2: Mass spectrometry during heating (no sample, $950 \mathrm{mbar} \mathrm{H}_{2}, 1010{ }^{\circ} \mathrm{C}$ reached at $16 \mathrm{~min}$ ) after a $\mathrm{GaP} / \mathrm{Si}(100)$ process. (a) Ga-related species. (b) P-related species.

Fig. 2 demonstrates that out-gassing of both $\mathrm{Ga}$ and $\mathrm{P}$ species from reactor parts needs to be considered: After a typcial GaP/Si growth run, we heated the reactor to $1010^{\circ} \mathrm{C}(950$ mbar $\mathrm{H}_{2}$ ) and monitored the ratio of atomic weights : degree of ionization, which relate to $\mathrm{P}$ and $\mathrm{Ga}$, with a MS connected to the reactor outlet. While we cannot directly translate the measured ionization currents to partial pressures in the reactor, both $\mathrm{Ga}, \mathrm{GaH}_{x}, \mathrm{P}$ and 
$\mathrm{PH}_{x}$ species are clearly present in the gas phase.

In order to study the effect of background residuals quantitatively, we performed XPS measurements. Coverages were quantitatively estimated applying a model described in Ref., ${ }^{14}$ including the Si 2 p plasmon loss peak. One ML would correspond to the thickness of a quarter of a lattice constant with every atomic substrate site replaced by the overlayer species. Here, we compare $\mathrm{Si}_{\text {clean }}$ to $\mathrm{Si}_{\text {cont }}$, where we increased the reactor pressure gradient after Si buffer growth. Both samples were transferred to XPS ${ }^{9}$ after $\operatorname{Si}(100)$ processing and prior to pulsed GaP nucleation. Fig. 3 shows selected PE lines and Tab.1 gives binding energies $\left(\mathrm{E}_{B}\right)$ and coverages. For both samples, the Ga 2p $\mathrm{p}_{3 / 2}$ photoemission (PE) line (Fig. 3(a)) clearly consists of two components: a bigger component $\left(\mathrm{Ga}_{2}\right)$ and a smaller one at lower binding energies $\left(\mathrm{Ga}_{1}\right)$. Compared to recently published data for a thicker $\mathrm{GaP} / \mathrm{Si}(100)$ reference, ${ }^{8}$ the entire spectrum is shifted towards higher binding energies as the Fermi level at the surface here is located closer to the conduction band. Considering that shift, $\mathrm{Ga}_{2}$ matches the position of GaP. Due to the small cross-section and lower surface-sensitivity, the P 2p PE line (Fig. 3(b))of $\mathrm{Si}_{\text {cont }}$ is not intense enough to reliably distinguish between the existence of two or just one component. ${ }^{(i)} \mathrm{Si}_{\text {cont }}$, however, is covered by about 9 times more $\mathrm{Ga}$ and about 2 times more $\mathrm{P}$ compared to $\mathrm{Si}_{\text {clean }}$. When $\mathrm{Si}_{\text {cont }}$ is measured at $30^{\circ}$ exit angle to raise surface sensitivity, the ratio $\mathrm{Ga}_{1}: \mathrm{Ga}_{2}$ increases, whereas $\mathrm{Ga}_{1}: \mathrm{P}$ remains constant (see Tab.1). ${ }^{\text {(ii) }}$ This implies that the $\mathrm{Ga}_{2}$ signal is more attenuated than that of $\mathrm{Ga}_{1}$ and $\mathrm{P}$. We interpret this as $\mathrm{Ga}_{2}$ being covered by another species, while $\mathrm{Ga}_{1}$ and $\mathrm{P}$ are not. One possible explanation is that three different adsorbate species coexist at the surface: (i) about $9 \%$ of a monolayer (ML) GaP, where $\mathrm{Ga}$ (the $\mathrm{Ga}_{2}$ component) is situated below P, (ii) about $8 \% \mathrm{ML}$ of P-species not bound to Ga, and (iii) about 1\% ML Ga-species (the $\mathrm{Ga}_{1}$ component) not bound to P. The Si 2p PE line (Fig. 3(c)) cannot sufficiently be

\footnotetext{
(i) The different ratio of $\mathrm{Ga}_{2}: \mathrm{P}$ between the samples could explain the shift of the $\mathrm{P} 2 \mathrm{p}$ line position, as the two chemically shifted components, one would expect, cannot be reliably resolved.

(ii) Intensity ratios were calculated using SPECS ASF factors assuming a homogeneous element distribution. For thin layers, this underrates PE lines at higher kinetic energies, which explains the different ratio when compared to the ML coverage.
} 
fitted by neither one single component, nor by two components, due to the low coverages. Consequently, the Si 2p PE line does not allow a clear conclusion on whether the Ga and the $\mathrm{P}$ species are chemically bound to Si. Position-dependent measurements at $90^{\circ}$ exit angle of $\mathrm{Si}_{\text {cont }}$ suggest a decreasing $\mathrm{Ga}: \mathrm{P}$ ratio in flow direction (rear: $\mathrm{Ga}_{1}: \mathrm{P}=0.14, \mathrm{Ga}_{2}: \mathrm{P}=0.36$; front: $\left.\mathrm{Ga}_{1}: \mathrm{P}=0.17, \mathrm{Ga}_{2}: \mathrm{P}=0.74\right)$ with higher amounts of $\mathrm{Ga}$ at the front and more $\mathrm{P}$ at the rear of the sample.
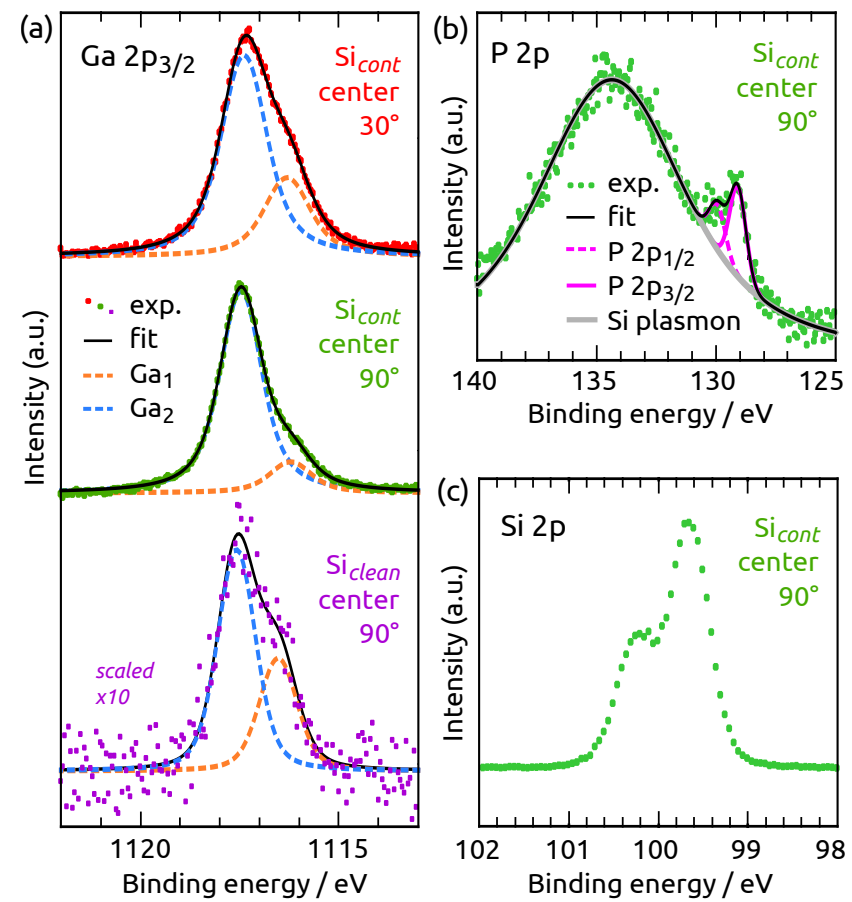

Figure 3: XPS measurements (monochromated $\mathrm{Al} \mathrm{K}_{\alpha}$ ). (a) Ga 2p $\mathrm{p}_{3 / 2} \mathrm{PE}$ line and fit components of $\mathrm{Si}_{\text {cont }}$ measured at normal exit angle (green) and $30^{\circ}$ exit angle (red) as well as of $\mathrm{Si}_{\text {clean }}$ (violet). (b) P $2 \mathrm{p} \mathrm{PE}$ line of $\mathrm{Si}_{\text {cont }}$ measured at normal exit angle and fit components including the Si 2p plasmon loss peak at $134.37 \mathrm{eV}(\mathrm{fwhm}=7.4 \mathrm{eV})$. (c) Si 2p PE line of $\mathrm{Si}_{\text {cont }}$ measured at normal exit angle.

Fig. 4 shows the RA spectra of one single $\mathrm{Si}_{\text {cont }}$ sample directly prior to nucleation as well as of $(2 \times 2) / c(4 \times 2)$ reconstructed $\mathrm{GaP} / \mathrm{Si}_{\text {cont }}$ after heteroepitaxy. The $\mathrm{Si}(100)$ signal (green, solid line) is similar to that of H-terminated $\mathrm{Si}(100)$, though there are slight differences: Beyond $3.9 \mathrm{eV}$, RAS of $\mathrm{Si}_{\text {cont }}$ does not clearly show the peak-like contribution known from clean H-terminated $\mathrm{Si}(100),{ }^{5,15}$ but an additional broad peak seems present around $2.2 \mathrm{eV}$. The clear peak at the $\mathrm{E}_{1}$ interband transition of Si, however, corresponds to a preferential A- 
Table 1: Coverage $\mathcal{C}$ in $\mathrm{ML},{ }^{14} \mathrm{E}_{B}$ (wrt. $\mathrm{E}_{F}$ ), intensity ratios (corrected with atomic senistivity factors $(\mathrm{ASF}))$ of the $2 \mathrm{p}_{3 / 2}$ PE components shown in Fig. 3

\begin{tabular}{c|rr|rr|r|rr}
\hline & \multicolumn{2}{|c|}{$\mathrm{Si}_{\text {clean }}$} & \multicolumn{6}{|c}{$\mathrm{Si}_{\text {cont }}$} \\
PE line & $\mathrm{E}_{B} / \mathrm{eV}$ & $\mathcal{C} / \mathrm{ML}$ & $\mathrm{E}_{B} / \mathrm{eV}$ & $\mathcal{C} / \mathrm{ML}$ & ratio & $90^{\circ}$ & $30^{\circ}$ \\
\hline $\mathrm{Ga}_{1}$ & 1116.53 & $0.4 \%$ & 1116.28 & $1.3 \%$ & $\mathrm{Ga}_{1}: \mathrm{Ga}_{2}$ & 0.17 & 0.39 \\
$\mathrm{Ga}_{2}$ & 1117.58 & $0.7 \%$ & 1117.48 & $8.6 \%$ & $\mathrm{Ga}_{1}: \mathrm{P}$ & 0.10 & 0.11 \\
$\mathrm{P}$ & 129.38 & $8 \%$ & 129.09 & $17 \%$ & $\mathrm{Ga}_{2}: \mathrm{P}$ & 0.68 & 0.28 \\
$\mathrm{Si}$ & 99.76 & - & 99.65 & - & & & \\
\hline
\end{tabular}

type Si dimer orientation, which is indicated in the inset (b). RA spectra of $(2 \times 2) / c(4 \times 2)$ reconstructed $\mathrm{GaP} / \mathrm{Si}(100)$ are related to the buckled $\mathrm{P}$ dimers and the sign of the RAS signal (at the surface-modified bulk transition below $\mathrm{E}_{1}$ of $\mathrm{GaP}$ and at the transition at about $2.5 \mathrm{eV}$ ) corresponds to the $\mathrm{P}$ dimer orientation. ${ }^{11-13,16}$ The $\mathrm{GaP} / \mathrm{Si}_{\text {cont }} \mathrm{RA}$ spectrum measured here (orange, dash-dotted line) corresponds to majority A-type P dimers. The intensity, however, indicates that antiphase disorder did not completely annihilate. ${ }^{13}$ Hence, in this case, RAS proves growth of majority A-type GaP on preferential A-type Si. Within an abrupt interface model, ${ }^{7,17}$ the case $\mathrm{A} \rightarrow \mathrm{A}$ implies $\mathrm{Si}-\mathrm{Ga}$ bonds at the heterointerface as indicated in the inset (c) of Fig. 4.

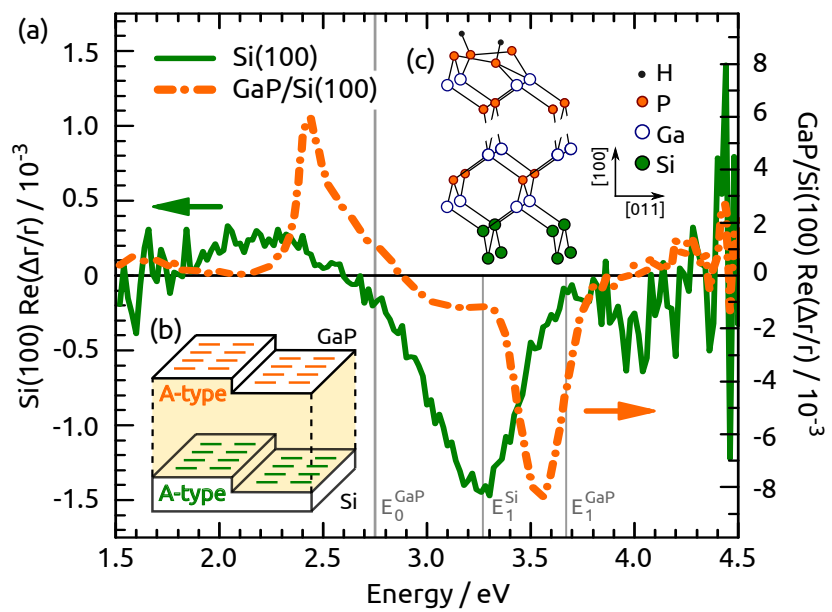

Figure 4: (a) RA spectra of $\mathrm{Si}_{\text {cont }}$ directly prior to $\mathrm{GaP}$ nucleation $\left(420^{\circ} \mathrm{C}\right)$ and of subsequently grown, $(2 \times 2) / c(4 \times 2)$ reconstructed $\mathrm{GaP}\left(50^{\circ} \mathrm{C}\right)$. (b) Corresponding orientations of $\mathrm{P}$ and Si dimers (prior to nucleation). (c) Sketch of the heterointerface in the abrupt model. Vertical grey lines indicate the critical point energies of $\mathrm{Si}^{18}$ and $\mathrm{GaP},{ }^{19}$ respectively. 
Both for $\mathrm{GaP}$ growth on $\mathrm{Si}_{\text {clean }}$ (see Fig. $1(\mathrm{a})$ ) and $\mathrm{Si}(100)$ with $2^{\circ}$ offcut, ${ }^{7}$ in contrast, the $\mathrm{GaP}$ sublattice orientation is inverted, which implies $\mathrm{Si}-\mathrm{P}$ bonds at the heterointerface. ${ }^{7}$ In principle, the inversion of the GaP sublattice could also be explained by $\mathrm{Si}-\mathrm{P}$ bonds combined with either (i) B-type $\mathrm{Si}(100)$, (ii) substitutional $\mathrm{P}$ adsorption or (iii) an additional Ga/P interlayer forming one ML below the uppermost Si atoms. However, these processes would then only occur during pulsed nucleation on $\mathrm{Si}_{\text {cont }}$ and not on $\mathrm{Si}_{\text {clean }}$. ${ }^{\text {(iii) }}$ We observed $\mathrm{Si}-\mathrm{P}$ bonds and a GaP sublattice orientation in disagreement with substitutional $\mathrm{P}$ adsorption of a whole Si ML in Ref. ${ }^{8}$ during GaP nucleation in comparably clean systems. In case of $\mathrm{Si}_{\text {cont }}$, our XPS analysis indicates that $\mathrm{P}$ is adsorbed on top of $\mathrm{Si}$. Consequently, it seems unlikely that $\mathrm{P}$ will substitute $\mathrm{Si}$ atoms during subsequent GaP nucleation. Ga was found to promote B-type $\operatorname{Si}(100)$ terraces at $\mathrm{Si}(100)$, which was Joule-heated to $600{ }^{\circ} \mathrm{C}$ in UHV. $^{22}$ To explain our findings, rearrangement towards Ga-covered B-type Si terraces ${ }^{22}$ would have to take place during pulsed nucleation simultaneously with replacement of the uppermost Ga atoms by $\mathrm{P}$, which we find unlikely. Ga droplet growth on $\mathrm{Si}(100)$ terraces with precursor supply may lead to pyramidal etching. ${ }^{23,24}$ The droplet formation was found to be significantly above $1 \mathrm{ML}$ of Ga and can be reduced by higher V:III ratios. ${ }^{25}$ Here, Ga coverages prior to nucleation are clearly below 1 ML and XPS results even imply that the larger $\mathrm{Ga}_{2}$ component, which is not located at the very surface, is bound to $\mathrm{P}$ whereas the $\mathrm{Ga}_{1}$ component is on top. Regarding Ga coverages below 0.5 ML, UHV studies suggest that Ga-Ga dimers form on top of Si dimers which remain unbroken. ${ }^{26-28}$ Though experimental conditions are quite different here (monohydride terminated $\mathrm{Si}$, presence of $\mathrm{P}$ ), intact A-type Si dimers below the adsorbed Ga could explain the $\mathrm{Si}$ dimer related RAS minimum at $\mathrm{E}_{1}^{\mathrm{Si}}$ (Fig. 4) and possibly also the slight differences in lineshape above and below $\mathrm{E}_{1}^{\mathrm{Si}}$ compared to $\mathrm{Si}(100)$ prepared in absence of III-V residuals. ${ }^{5}$ The increased amount of Ga available during GaP nucleation also shifts the (P,Ga) chemical potential towards more Ga-rich conditions. $A b$

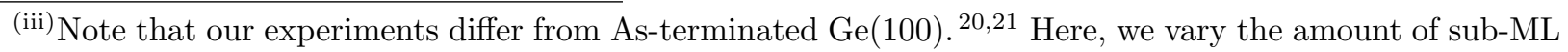
residuals on Si with A-type majority domains. Subsequently, pulsed nucleation with precursor supply is performed identically.
} 
initio density functional theory calculations showed that the energetically favorable binding situation at the $\mathrm{GaP} / \mathrm{Si}(100)$ heterointerface changes from $\mathrm{Si}-\mathrm{P}$ to $\mathrm{Si}-\mathrm{Ga}$ for more Ga-rich nucleation conditions, ${ }^{7}$ which can explain the observation of inverted GaP sublattice growth on $\mathrm{Si}_{\text {clean }}$ resp. $\mathrm{Si}_{\text {cont }}$. It may also explain the $\mathrm{Si}-\mathrm{Ga}$ bonds suggested recently. ${ }^{17}$

We believe that the Si surface preparation step after Si buffer growth is decisive here for the amount of residuals found on the surface. Annealing at $730{ }^{\circ} \mathrm{C}$ in $950 \mathrm{mbar}_{2}$ after thermal deoxidation at $1000^{\circ} \mathrm{C}$ is crucial for single-domain, A-type surface preparation of $\operatorname{Si}(100) 2^{\circ} \cdot{ }^{5}$ Quick temperature and pressure ramps, however, are necessary to avoid layer-bylayer removal on $\mathrm{Si}(100) 0.1^{\circ}$ occuring in this temperature range. ${ }^{6}$ Particularly the pressure ramp is complicating $\mathrm{Si}(100) 0.1^{\circ}$ surface preparation in reactors contaminated with IIIV residuals: The rate of residuals desorbing from reactor parts (liner, susceptor, carrier) is increasing with decreasing pressure. This becomes particularly important at elevated temperatures, where desorption of residuals from reactor parts and diffusion of residuals on the sample are high while the $\mathrm{H}$ termination ${ }^{2}$ is not stable yet. The consequence is that single-domain surface formation and avoidance of contamination conflict for nominal $\mathrm{Si}(100)$ substrates regarding process parameters. The residual atoms may also influence Si vacancy generation and diffusion, but in situ RAS reveals an A-type majority domains even for $\mathrm{Si}_{\text {cont }}$ so that we believe that the kinetic surface processes are comparable to Ref. ${ }^{6}$ Nucleation with a starting TEGa pulse (not shown here) was not sufficient to grow GaP with majority A-type P dimers. Nucleation with high residual amounts or high TEGa precursor supply-in order to reach high amounts of Ga for single-domain GaP-is not easy to control. The co-existence of areas with $\mathrm{Si}-\mathrm{P}$ and $\mathrm{Si}-\mathrm{Ga}$ bonds at single-domain $\mathrm{Si}(100)$ terraces, however, would cause antiphase disorder analogously to the existence of either $\mathrm{Si}-\mathrm{P}$ or $\mathrm{Si}-$ Ga bonds at two-domain $\mathrm{Si}(100)$ terraces. We never observed A-type $\mathrm{GaP} / \mathrm{Si}(100)$ surfaces in case of A-type $\operatorname{Si}(100) 2^{\circ}$ misoriented substrates. Cooling to temperatures below $730^{\circ} \mathrm{C}$ in $950 \mathrm{mbar}_{2}$ (for annealing to prepare A-type terraces) prior to decreasing the reactor pressure for GaP nucleation seems to effectively hinder excessive Ga diffusion on the surface. 
Both decreased desorption rates from reactor parts and the $\mathrm{H}$ termination of the $\mathrm{Si}(100)$ surface $^{2}$ are benificial here. Nucleation on nominally oriented $\mathrm{Si}(100)$ may also benefit from even higher TBP partial pressures.

In conclusion, we showed that a compromise between the avoidance of layer-by-layer removal and diffusion of $(\mathrm{Ga}, \mathrm{P})$ residuals to the substrate must be made for $\mathrm{Si}(100)$ surface preparation. Sub-monolayer coverages of $(\mathrm{Ga}, \mathrm{P})$ residuals on the $\mathrm{Si}(100)$ surface-prior offering precursors for pulsed nucleation-strongly influence GaP nucleation. The majority GaP sublattice orientation changes for higher amounts of Ga present at the surface. We explain this by a dependency of abrupt heterointerface structures on the chemical potential during nucleation ${ }^{7}$ and the presence of $\mathrm{Si}-\mathrm{Ga}$ nucleation seeds prior to offering the first precursor pulse. The coexistence of $\mathrm{Si}-\mathrm{P}$ and $\mathrm{Si}-\mathrm{Ga}$ bond domains can lead to antiphase disorder in the GaP epilayer. Consequently, the residual background pressure needs to be controlled precisely for well defined interface preparation. Time-resolved studies are planned in order to resolve the optical anisotropy of the presumably Si-Ga heterointerfaces.

It is a pleasure to thank W. Daum, G. Lilienkamp, B. Borkenhagen, F. Grosse, and O. Romanyuk for valuable discussions. This work was financially supported by the DFG (proj. no. HA3096/4-1).

\section{References}

(1) Kroemer, H. Polar-on-Nonpolar Epitaxy. J. Cryst. Growth 1987, 81, 193-204.

(2) Brückner, S.; Döscher, H.; Kleinschmidt, P.; Hannappel, T. In Situ Investigation of Hydrogen Interacting with Si(100). Appl. Phys. Lett. 2011, 98, 211909.

(3) Chadi, D. J. Stabilities of Single-Layer and Bilayer Steps on Si(001) Surfaces. Phys. Rev. Lett. 1987, 59, 1691-1694.

(4) Laracuente, A. R.; Whitman, L. J. Step Structure and Surface Morphology of HydrogenTerminated Silicon: (001) to (114). Surf. Sci. 2003, 545, 70-84. 
(5) Brückner, S.; Döscher, H.; Kleinschmidt, P.; Supplie, O.; Dobrich, A.; Hannappel, T. Anomalous Double-Layer Step Formation on Si(100) in Hydrogen Process Ambient. Phys. Rev. B 2012, 86, 195310.

(6) Brückner, S.; Kleinschmidt, P.; Supplie, O.; Döscher, H.; Hannappel, T. DomainSensitive In Situ Observation of Layer-by-Layer Removal at $\mathrm{Si}(100)$ in $\mathrm{H}_{2}$ Ambient. New J. Phys. 2013, 15, 113049.

(7) Supplie, O.; Brückner, S.; Romanyuk, O.; Döscher, H.; Höhn, C.; May, M. M.; Kleinschmidt, P.; Grosse, F.; Hannappel, T. Atomic Scale Analysis of the GaP/Si(100) Heterointerface by in situ Reflection Anisotropy Spectroscopy and ab initio Density Functional Theory. Phys. Rev. B 2014, 90, 235301.

(8) Supplie, O.; May, M. M.; Steinbach, G.; Romanyuk, O.; Grosse, F.; Nägelein, A.; Kleinschmidt, P.; Brückner, S.; Hannappel, T. Time-Resolved Optical In Situ Spectroscopy During Formation of the GaP/Si(100) Heterointerface. J. Phys. Chem. Lett. 2015, 6, 464.

(9) Hannappel, T.; Visbeck, S.; Töben, L.; Willig, F. Apparatus for Investigating Metalorganic Chemical Vapor Deposition-Grown Semiconductors with Ultrahigh-Vacuum based Techniques. Rev. Sci. Instrum. 2004, 75, 1297-1304.

(10) Aspnes, D. E.; Studna, A. A. Anisotropies in the Above-Band-Gap Optical Spectra of Cubic Semiconductors. Phys. Rev. Lett. 1985, 54, 1956-1959.

(11) Töben, L.; Hannappel, T.; Möller, K.; Crawack, H.; Pettenkofer, C.; Willig, F. RDS, LEED and STM of the P-rich and Ga-rich Surfaces of GaP(100). Surf. Sci. 2001, 494, L755-L760.

(12) Hahn, P. H.; Schmidt, W. G.; Bechstedt, F.; Pulci, O.; Sole, R. D. P-rich $\operatorname{GaP}(001)(2 \times 1) /(2 \times 2)$ Surface: A Hydrogen-Adsorbate Structure Determined from First-Principles Calculations. Phys. Rev. B 2003, 68, 033311. 
(13) Döscher, H.; Hannappel, T. In Situ Reflection Anisotropy Spectroscopy Analysis of Heteroepitaxial GaP Films Grown on Si(100). J. Appl. Phys. 2010, 107, 123523.

(14) May, M.; Lewerenz, H.-J.; Hannappel, T. Optical In Situ Study of InP(100) Surface Chemistry: Dissociative Adsorption of Water and Oxygen. J. Phys.Chem. C 2014, $118,19032-19041$.

(15) Palummo, M.; Witkowski, N.; Pluchery, O.; Del Sole, R.; Borensztein, Y. ReflectanceAnisotropy Spectroscopy and Surface Differential Reflectance Spectra at the Si(100) Surface: Combined Experimental and Theoretical Study. Phys. Rev. B 2009, 79, 035327 .

(16) Sippel, P.; Supplie, O.; May, M. M.; Eichberger, R.; Hannappel, T. Electronic Structures of GaP(100) Surface Reconstructions Probed with Two-Photon Photoemission Spectroscopy. Phys. Rev. B 2014, 89, 165312.

(17) Beyer, A.; Ohlmann, J.; Liebich, S.; Heim, H.; Witte, G.; Stolz, W.; Volz, K. GaP Heteroepitaxy on Si(001): Correlation of Si-Surface Structure, GaP Growth Conditions, and Si-III/V Interface Structure. J. Appl. Phys. 2012, 111, 083534.

(18) Lautenschlager, P.; Garriga, M.; Vina, L.; Cardona, M. Temperature Dependence of the Dielectric Function and Interband Critical Points in Silicon. Phys. Rev. B 1987, 36,4821 .

(19) Zollner, S.; Garriga, M.; Kircher, J.; Humlícek, J.; Cardona, M.; Neuhold, G. Temperature Dependence of the Dielectric Function and the Interband Critical-Point Parameters of GaP. Thin Solid Films 1993, 233, 185-188.

(20) Brückner, S.; Supplie, O.; Barrigón, E.; Luczak, J.; Kleinschmidt, P.; Rey-Stolle, I.; Döscher, H.; Hannappel, T. In Situ Control of As Dimer Orientation on Ge(100) Surfaces. Appl. Phys. Lett. 2012, 101, 121602. 
(21) Ting, S. M.; Fitzgerald, E. A. Metal-Organic Chemical Vapor Deposition of Single Domain GaAs on Ge/Ge $\mathrm{Ge}_{x} \mathrm{Si}_{1 x} / \mathrm{Si}$ and Ge Substrates. J. Appl. Phys. 2000, 87, 26182628.

(22) Hara, S.; Irokawa, K.; Miki, H.; Kawazu, A.; Torii, H.; Fujishiro, H. I. Behavior of Ga atoms on Si(001) Surface at High Temperature. J. Appl. Phys. 2005, 98, 0835130835137.

(23) Bell, K. A.; Ebert, M.; Yoo, S. D.; Flock, K.; Aspnes, D. E. Real-Time Optical Characterization of Heteroepitaxy by Organometallic Chemical Vapor Deposition. J. Vac. Sci. Technol. A 2000, 18, 1184-1189.

(24) Werner, K.; Beyer, A.; Oelerich, J.; Baranovskii, S.; Stolz, W.; Volz, K. Structural Characteristics of Gallium Metal Deposited on Si(001) by MOCVD. J. Cryst. Growth 2014, 405, 102-109.

(25) Volz, K.; Beyer, A.; Witte, W.; Ohlmann, J.; Németh, I.; Kunert, B.; Stolz, W. GaPNucleation on Exact Si(001) Substrates for III/V Device Integration. J. Cryst. Growth 2011, 315, 37-47.

(26) Bourguignon, B.; Carleton, K. L.; Leone, S. R. Surface Structures and Growth Mechanism of Ga on Si(100) Determined by LEED and Auger Electron Spectroscopy. Surf. Sci. 1988, 204, 455-472.

(27) Baski, A. A.; Nogami, J.; Quate, C. F. Gallium Growth and Reconstruction on the Si(100) Surface. J. Vac. Sci. Technol. A 1990, 8, 245-248.

(28) Sakama, H.; Kawazu, A.; Sueyoshi, T.; Sato, T.; Iwatsuki, M. Scanning Tunneling Microscopy on Ga/Si(100). Phys. Rev. B 1996, 54, 8756-8760. 


\section{Graphical TOC Entry}

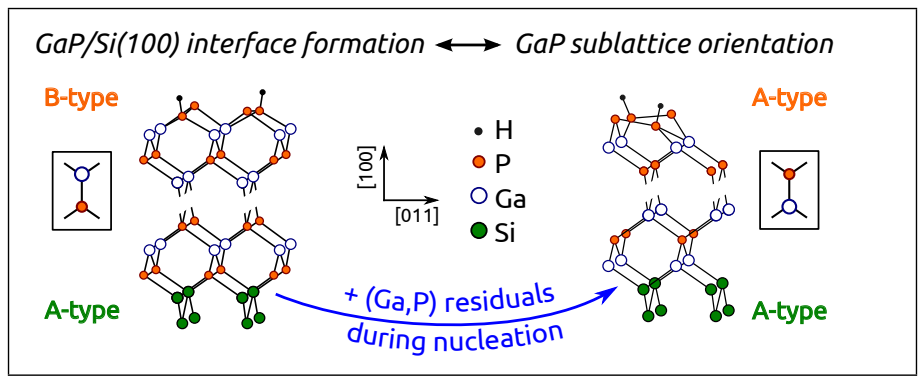

